University of Nebraska - Lincoln

DigitalCommons@University of Nebraska - Lincoln

French Language and Literature Papers

Modern Languages and Literatures, Department

2021

[Lydie Salvayre,] Dans le vif du vivant

Warren Motte

Follow this and additional works at: https://digitalcommons.unl.edu/modlangfrench

Part of the French and Francophone Language and Literature Commons

This Article is brought to you for free and open access by the Modern Languages and Literatures, Department of at DigitalCommons@University of Nebraska - Lincoln. It has been accepted for inclusion in French Language and Literature Papers by an authorized administrator of DigitalCommons@University of Nebraska - Lincoln. 


\section{Warren Motte}

\section{Dans le vif du vivant}

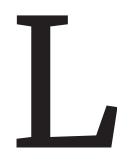

es livres de Lydie Salvayre sont implacables, obsessionnels, insistants. Ils débordent de gens qui hurlent, qui fustigent, qui accusent, des gens qui estiment qu'il y a quelque chose qui ne va pas dans l'ordre accepté des choses, et qui ne relâcheront pas. Salvayre dit « Non! » de manière superbement éloquente ; elle refuse d'approuver la sagesse reçue; elle pense que tout mérite d'être interrogé. À notre époque si houleuse et incertaine, ces livres sont vivifiants, des vaccins puissants contre les foutaises outrageuses qui nous bombardent de façon quotidienne, constante. Son écriture se situe dans le vif du vivant, pour emprunter une phrase de Salvayre elle-même, phrase dont elle se sert pour décrire la façon d'être de Pablo Picasso. Vif comme vital, le site où tous les sens sont activés, où rien ne passe inaperçu, où notre attention se focalise et où le jeu de la vie se déroule de manière débridée. Vif comme rapide, ardent, sensible, intense, réactif, aigu. Vif comme listo aussi, ce mot espagnol qui désigne l'empressement, mais qui connote également l'alacrité intellectuelle, l'habilité, la volonté d'agir immédiatement. Ce mot de la langue maternelle de Salvayre lui va comme un gant, parce qu'elle est surtout lista : rapide à réagir, rapide à comprendre, rapide à s'engager avec nous, rapide à demander notre engagement avec elle.

Published in Lydie Salvayre, maintenant même, ed. Warren Motte (Lincoln, NE : Zea Books, 2021). doi: 10.32873/unl.dc.zea.1281 
Lydie Salvayre n'est pas née dans la littérature française ; au contraire, elle y est venue selon ses propres termes. Ses parents étaient des républicains espagnols qui avaient gagné la France en 1939, à la fin de la Guerre Civile, lorsque les dernières forces républicaines cédaient sous l'assaut des fascistes. Son père était andalou et communiste ; sa mère catalane avait été fortement impressionnée par l'anarchisme actif et pragmatique à Barcelone. Ils se sont rencontrés dans le camp d'internement notoire d'Argelès. Née en 1948 sous le nom de Lydie Arjona, Salvayre a grandi dans une communauté de réfugiés près de Toulouse, où selon ses propres dires le débat politique était son pain quotidien, et le souvenir de la Guerre Civile demeurait frais, bien après sa fin. Jusqu'à sa rentrée dans l'école primaire, où elle a été obligée d'apprendre le français, elle ne parlait que l'espagnol. Elle a commencé à lire avidement au lycée, et elle a décroché une licence de littérature à l'université de Toulouse. Ayant découvert la psychanalyse, elle s'est inscrite à la faculté de médecine, où elle a obtenu un autre diplôme. Elle est devenue psychiatre, se spécialisant éventuellement dans la psychiatrie pédagogique. Elle a publié son premier roman, La Déclaration, en 1990.

Depuis lors, elle a écrit une vingtaine de livres, et elle s'est fait une place sur l'horizon de la littérature française contemporaine. Parmi les prix qu'elle a reçus, on citera le Goncourt décerné en 2014 à Pas pleurer, roman qui reprend l'éveil politique, culturel et sexuel de sa mère, adolescente à l'époque découvrant Barcelone en pleine révolution. Le Goncourt est sans doute le plus prestigieux parmi les nombreux prix littéraires français, et cela fournit une vaste considération à l'auteur qui le reçoit, car il assure quelque chose comme un demi-million de ventes, ainsi qu'un attention médiatique énorme. Cependant, malgré toute cette 
reconnaissance, on a l'impression que Salvayre est moins confortable sur un piédestal que dans les marges, dans une posture d'interrogation, exerçant une ironie finement affûtée afin de mettre en question la pensée conventionnelle et les canons qui l'articulent. Ceux qui la lisent attentivement noteront un scepticisme à l'égard des établissements de différentes sortes - y compris l'établissement littéraire-ainsi qu'une affirmation de sa propre particularité, des effets qui parcourent son œuvre depuis le début jusqu'au présent.

J'aimerais survoler cet œuvre très rapidement, à l'intention de ceux qui ne le connaissent pas, ou peu. Ce faisant, j'essayerai de mettre en lumière certains traits clefs de son écriture, ceux qui me semblent signifiants. L'importance qu'elle accorde à la voix, par exemple, sa façon de traduire la voix dans ses romans, sa manière d'écouter des voix très variées, sa manière de nous encourager à écoutermême si nous sommes les seuls qui écoutons. Je suis impressionné aussi par sa façon de penser le langage, l'imaginant en termes relativistes, comme tout individu bilingue doit le faire, plutôt qu'en termes absolus, comme les gens monolingues. Son emploi de l'ironie mérite notre attention, parce que l'ironie est un outil dangereux qui peut se retourner contre la personne qui la déploie, pour peu que la vigilance de cette personne se relâche. Il convient aussi de souligner la manière dont Salvayre met la société française en procès dans ses livres, obligeant son lecteur à considérer certaines caractéristiques de cette société que la littérature plus conventionnelle passe sous silence. Mais ce qui m'intéresse le plus, peut-être, c'est l'esprit anticonformiste qui anime l'écriture de Salvayre. Elle pratique ce que Ross Chambers appelle « conduite oppositionnelle », élaborant des stratégies de résistance qui exploitent les points faibles 
des structures du pouvoir, retournant le pouvoir contre luimême par un geste radicalement critique.

Tous ces traits figurent dans La Déclaration (1990). Comme beaucoup de ses romans, c'est un texte monologique où l'unique voix parle implacablement. Le protagoniste est un homme abandonné par son épouse, et qui se sent absolument seul dans le monde. Personne ne l'écoute, il se plaint, personne ne se soucie de lui, les gens le fuient comme si sa solitude était contagieuse. Il se dégoûte de lui-même et de sa situation misérable dans la vie, la force du réel l'écrase, il est incapable d'humour, d'aise ou de grâce. Il soupçonne que la folie l'attend. Bref, c'est un individu en crise-et c'est cela, plus que toute autre chose, qui intéresse Salvayre. Il en est venu au point où la seule chose à faire, c'est de hurler son désespoir. De toute évidence, Salvayre estime qu'un tel hurlement signifie pleinement, et que cela mérite notre attention, aussi difficile que cela peut être de l'écouter.

Salvayre nous offre une invitation similaire dans La Vie commune (1991). La narratrice du roman s'appelle Suzanne, une femme mûre qui travaille comme secrétaire dans une entreprise. Elle nourrit une haine prononcée pour une collègue nouvellement arrivée. Tout de cette collègue agace $\mathrm{Su}-$ zanne : elle fume, elle mâche du chewing-gum, et la profondeur de son hypocrisie n'a d'égal que la profondeur de son décolleté. Suzanne brûle d'une rage qui se retourne contre elle-même de façon inéluctable, car comme le narrateur de La Déclaration, elle ne peut rien voir en dehors de cette rage. La Vie commune est une parabole d'obsession qui trace la manière dont les irritations ordinaires, intériorisées et suppurantes, peuvent assumer des proportions massives dans l'esprit de quelqu'un qui cherche la catastrophe, le désastre, le drame-qui cherche n'importe quoi pour fournir un peu de couleur à une existence absolument monochrome. 
Dans La Médaille (1993) Salvayre donne libre cours à son penchant pour l'ironie. Elle mise sur la voix encore une fois, mais ici plusieurs voix rentrent en jeu, plutôt qu'une seule. L'occasion est une cérémonie de fin d'année dans une usine, où les patrons font l'éloge de ceux parmi les ouvriers désignés comme spécialement méritants, et ces ouvriers rendent la pareille, exprimant leur gratitude face à cette reconnaissance inespérée. L'économie discursive est déséquilibrée en faveur des patrons, comme on pourrait s'y attendre. D'une part, les patrons ont l'habitude d'employer le langage comme une technique de pouvoir et de contrôle. D'autre part, ils étouffent rapidement toute tentative de la part des ouvriers à employer le langage de façon autre que purement phatique. On apprend (par inférence, pour la plupart) que des perturbations couvent au sein de l'usine : certains ouvriers sont désaffectés, certains des lauréats sont des indicateurs, la grève se profile. Les patrons savent qu'il faut que leurs mots comptent, et donc ils les déploient comme des sabres. Le langage dans ce roman est lacérant : il coupe et il tranche.

Un autre bavard occupe le centre de la scène dans La Puissance des mouches (1995). Il est gardien de musée à PortRoyal-des-Champs, l'abbaye à côté de Paris où Blaise Pascal s'était retiré. Le gardien de musée est un homme qui a beaucoup souffert : c'est le fils d'un père brutal, sa femme l'a quitté, son patron le maltraite cruellement. Quelque part en chemin il a tué quelqu'un, mais il nous faudra attendre la fin du roman pour savoir qui. La prison où il vit maintenant, et d'où il nous parle, sert simplement à matérialiser les conditions carcérales de sa vie d'avant son inculpation. Le monde que Salvayre construit dans ce roman est exceptionnellement exigu. Il n'y a pas de sortie, pas de place pour se retourner ; c'est un espace claustral et angoissant 
où les possibilités sont extrêmement contraintes, réduites et inévitables.

Située dans la banlieue ouvrière de Paris, La Compagnie des spectres (1997) est l'histoire d'une femme qui s'appelle Rose, de sa fille de dix-huit ans, Louisiane, et d'un huissier nommé Maître Échinard, venu les expulser de leur appartement. À travers Rose, Salvayre engage l'historique et la politique plus largement que dans ses romans précédents, parce que Rose vit simultanément dans le présent et dans le passé-ce passé étant les années de l'Occupation allemande, pendant lesquelles sont frère s'était fait assassiner par la milice française collaborationniste. Rose est persuadée que Maître Échinard a été envoyé par Joseph Darnand (le chef de la milice, traduit en justice et exécuté pour ses crimes peu après la Libération), et rien ne peut l'en dissuader. Elle est folle, certainement, mais sa folie sert à mettre en évidence certaines iniquités de la société française qui n'ont pas beaucoup changé dans le demi-siècle depuis la fin de la guerre.

Dans Quelques Conseils utiles aux élèves huissiers (1997) Maître Échinard revient, cette fois parlant de sa propre voix, et à pleine gorge. Il s'adresse à une salle bondée d'apprentis, décrivant les situations qu'ils risquent de rencontrer une fois qu'ils seront pleinement en possession de leurs titres professionnels. Il évoque sa rencontre avec Rose et Louisiane comme étude de cas exemplaire, mais son récit est bien différent de celui que Salvayre nous offre dans La Compagnie des spectres. Hélas, ses élèves sont terriblement obtus. Ils n'ont jamais entendu parler de Darnand, ils ne savent pas ce que cela veut dire, le mot paranoïa. Maître Échinard est donc obligé de procéder de manière très lente et judicieuse s'il espère partager une portion, même infime, de sa sagesse avec ces élèves. Mais étant donné le métier qu'il exerce, il a naturellement l'habitude des gens aussi stupides qu'insipides. 
Comme dans certains de ses autres textes, Lydie Salvayre nous invite ici à lire contre le grain du discours de son narrateur, afin d'apprécier des réalités qu'il est lui-même incapable de reconnaître.

Dans La Conférence de Cintegabelle (1999) un homme fait une conférence sur les vertus de la conversation devant un public d'amis et voisins dans un village pyrénéen. Son épouse, apprend-on, est morte il y a deux mois - et nous apprendrons beaucoup plus à son sujet au cours du récit, mais seulement par bribes. La conversation est un art, selon le conférencier, un art éminemment français. Pourtant, il craint le déclin de cet art, et par conséquent le déclin du pays où il trouve son expression la plus noble. Ce qu'il propose aux âmes intrépides de Cintegabelle venues l'écouter, ce n'est rien de moins qu'un projet de revitalisation conversationnelle, et donc nationale. Mais la cause qu'il plaide avec tant d'éloquence en faveur de la conversation est implacablement monologique. C'est la première et la plus énorme ironie, parmi toutes celles qui circulent dans ce texte, ironies qui s'interrogent et se complémentent, qui demandent notre attention, qui nous amusent et nous exaspèrent, qui nous tiennent sur un pied d'alerte.

Différentes cultures se heurtent dans Les Belles Âmes (2000), qui prend comme sujet un reality tour dans lequel des touristes bourgeois bien-pensants visitent certains des bas quartiers les plus sinistrés d'Europe, afin de mieux apprécier comment vivent les pauvres. On pourrait s'attendre à ce qu'ils soient dévastés par la misère qu'ils découvrent, ou tout au moins un peu inquiétés. Mais leur vision du monde demeure relativement intacte et inchangée, et c'est leur bienveillance même qui sert à les protéger d'une vraie appréciation des réalités sociales, lorsque ces réalités sont trop affligeantes. Pendant leur voyage, de Paris à Bruxelles, à 
Cologne, à Berlin, à Dresde, à Milan et Turin, ces réalités deviennent de plus en plus rudes, car les organisateurs du tour ont délibérément programmé les choses ainsi, souhaitant que les touristes rencontrent des exemples de catastrophe sociale de plus en plus dramatiques. Pourtant, les mécanismes de défense psychologique des touristes se déclarent à la hauteur des circonstances, et les remparts qu'ils ont construits autour de leur conscience morale et politique sont aussi lisses et raffinés que l'est cet étonnant roman lui-même.

Le Vif du vivant (2001), dont j'emprunte le titre ici, est un billet d'amour de Lydie Salvayre à Pablo Picasso. Dans un long essai sur les carnets de Picasso de 1964, Salvayre médite une multiplicité de choses : la notion de l'exil, par exemple, et les contraintes de vivre dans un pays qui n'est pas le sien ; la liberté et ses conditions de possibilité ; le rôle de l'artiste dans la société ; le principe de l'excès dans la créativité ; les modes différents de l'engagement politique. Picasso est une figure exemplaire pour Salvayre. Elle admire son énergie artistique, la franchise de son regard, l'originalité de sa vision. Plus que toute autre chose, elle est impressionnée par la manière dont son art plaide pour la vie telle qu'elle est, sans compromis, et par sa façon de nous obliger de regarder les choses de manière directe et sans réserve. De toute évidence, Salvayre aspire à un même esprit dans son propre travail, et dans ce sens Le Vif du vivant nous en dit long sur sa propre conception de l'art, et sur sa manière de se positionner en tant qu'artiste.

Écrit dans le style élégamment mordant qui est devenu sa signature, Contre (2002) est une condamnation intraitable de la société française contemporaine, centrée sur l'idée de la complaisance culturelle, politique et idéologique. Salvayre parle dans sa propre voix ici, ayant laissé tomber l'illusion 
que fournit la fiction. À l'origine une commande de France Culture pour les rencontres de La Chartreuse du Festival d'Avignon, spectacle pour poète et deux guitares, la première a eu lieu au mois de juillet 2001. La version imprimée du texte est complétée par un CD audio de la performance, avec Salvayre qui lit son texte, accompagnée des guitaristes Serge Teyssot-Gay et Marc Sens. Peu des contradictions de la société française échappent au regard de Salvayre ici. Elle parcourt les registres personnels et politiques, individuels et collectifs. Elle dénonce la façon dont les médias exploitent l'imagination sanguinaire du public, créant ainsi un climat de la peur. Elle souligne comment les défavorisés et les figures marginales de toutes sortes, surtout les pauvres, se trouvent diabolisés. Elle déplore la commercialisation de la culture contemporaine, suggérant que la seule musique valorisée par cette culture est celle des cours boursiers. Elle tourne en dérision la manière où ses concitoyens se dopent de romans sentimentaux et de croissants au beurre, quand ils ne sont pas en train de se gaver de drogues psychotropiques, sous les meilleurs conseils médicaux. Elle se plaint d'une société où les amants se retrouvent par email seulement, et où la sexualité doit être avant tout sans risque. Afin de résister à de telles tendances, elle estime que nous devons agir, et que notre action doit être fondamentalement oppositionnelle, un geste incendiaire qui commence par le mot « Non!».

Et que les vers mangent le bœuf mort (2002) réunit sept textes relativement courts d'inspiration diverse. Il y a des méditations sur des dynamiques familiales ; il y a une pièce où des couples dansant un tango se disputent ; il y a même un essai lapidaire et convaincant en faveur de la paresse. Des versions de Quelques Conseils utiles aux élèves huissiers et Le Vif du vivant figurent ici également. Dans une note accompagnant ces textes, Salvayre nous invite à les imaginer comme 
des êtres habitant les marges de ses romans, et de considérer leur caractère « indiscipliné, déviant, disparate » comme une vertu plutôt qu'un défaut.

Passage à l'ennemie (2003) raconte l'histoire d'Adrien Arjona, policier chargé d'infiltrer un groupe de jeunes délinquants dans une cité de la banlieue parisienne. Les rapports qu'il envoie à ses supérieurs, formulés dans une prose riche et richement amusante, font la chronique d'une quête qui dérape de façon tout à fait plaisante. Obligé de fumer des quantités impressionnantes de haschisch afin de préserver sa clandestinité parmi les « déviants sociaux » qu'il doit surveiller, Arjona sera vite obligé de reconsidérer son propre système de valeurs. Il trouve les membres du groupe curieusement simpáticos, individuellement et collectivement. Et surtout la mystérieuse, silencieusement séductrice Dulcinée, une jeune femme encombrée d'un passé lourd. Leurs crimes sont au plus des peccadilles, le genre d'aventure que tout adolescent essaie, et Arjona se demande pourquoi les autorités ont désigné ces jeunes gens comme étant particulièrement dangereux. Contre son gré, il en vient à soupçonner qu'une telle désignation est infondée, et même pernicieuse. Peut-être que le pouvoir doit créer des ennemis là où il n'y en a pas, afin de s'affirmer et de projeter le spectacle de son hégémonie. Cette considération, ainsi que d'autres tout aussi subversives, conduira éventuellement Arjona à commettre une sorte d'autodafé idéologique. Pire encore, écrivant rapport après rapport, Arjona en vient à réaliser qu'il adore écrire. Et peut-être, après avoir mené sa mission au bout, écrira-t-il un roman la racontant. Dans le monde que Lydie Salvayre nous propose ici, une telle issue n'est pas impossible, car c'est un monde qui favorise la métamorphose et la transformation, un lieu où par la force de leur volonté, les cochons se font pousser des ailes et prennent leur envol. 
Le narrateur de La Méthode Mila (2005) est un célibataire d'une quarantaine d'années qui s'appelle Fausto Arjona. C'est un homme solitaire et misanthrope qui s'est retiré de la compagnie de ses semblables et qui passe ses journées, agréablement, à lire la philosophie et à regarder des films pornographiques. Cette idylle prend fin pourtant lorsque sa mère, veuve, vient aménager chez lui. S'occupant d'elle, Arjona voit la mort inscrite un peu partout sur son corps vieillissant, ce qui lui fait horreur. Progressivement, il en vient à abominer sa mère, découvrant dans cette abomination un côté monstrueux de lui-même qu'il n'avait jamais soupçonné. Il cherche consolation dans le Discours de la méthode de Descartes, n'en trouvant point. Arjona ressent le besoin d'une tout autre méthode, et il en trouvera une dans les démarches de Madame Mila, clairvoyante qui lui parle de sa putative ascendance en Andalousie au douzième siècle, le nourrissant d'un ragoût généalogique pimenté de Musulmans, de Chrétiens et de Juifs. Elle lui fournit ainsi une histoire-et encore plus important, un récit finement adapté à ses besoins. La Méthode Mila met en scène une lutte ironique entre philosophie et fiction, et la façon dont Lydie Salvayre choisit de résoudre ce conflit en dit long sur sa foi en le roman comme forme culturelle.

Dis pas ça (2006) nous offre une expérience dans la veine de Contre, et la version imprimée ressemble de près à celui-ci, dans la mesure où le texte s'accompagne encore une fois d'un enregistrement CD où Salvayre lit son texte, accompagnée encore de Serge Teyssot-Gay et Marc Sens, cette fois avec le bassiste Jean-Paul Roy. Encore comme Contre, la pièce a été présentée au Festival d'Avignon. Mais la similarité la plus frappante, c'est celle du ton : Dis pas ça est un exercice de négation radicale. Dans une série de dix vignettes très brèves, Salvayre dénonce une multiplicité de scandales, 
concernant des secrets familiaux jalousement gardés, la manière dont les médias exploitent l'image de la femme, ou la façon dont la droite politique encourage le racisme. Sa voix est intransigeante, son regard indéfectible, et la manière dont elle met en lumière les gestes qu'elle estime scandaleux ne laisse aucune marge de négociation possible.

Dans Portrait de l'écrivain en animal domestique (2007), Salvayre nous donne un animal qui sait faire plaisir à son maître, faisant des cabrioles et prenant des postures soumises afin de gagner la faveur de ce dernier. Cette bête, c'est la narratrice elle-même, qui conclut un pacte faustien avec Jim Tobold, le soi-disant « roi du hamburger » dont l'empire fast-food a consommé le monde entier, tout comme le monde entier consomme ses hamburgers. Son job, c'est d'écrire sa vie : pas une simple biographie, mais plutôt un testament, un Évangile de Tobold où le principe du capitalisme sauvage sera enfin élevé jusqu'au rang divin qu'il mérite, grâce aux efforts de son seul vrai prophète. Plus qu'un scribe, la narratrice fonctionne comme un miroir dans lequel Tobold admire sa propre personne. Elle s'assied à ses pieds dans un état d'extase alors qu'il explique qui il a été, qui il est et qui il sera. Tobold respire l'air raréfié d'un individu dont la fortune se moque de la notion de la richesse. Citoyen du monde à l'heure qu'il est, bien loin de ses origines modestes en France, il est constamment en mouvement, voltigeant de continent en continent, prenant un de ses nombreux Gulfstream comme un être inférieur prendrait un taxi. Malgré elle, la narratrice trouve qu'un tel mode de vie-aussi cruellement philistin que cela aurait pu lui sembler avant l'abandon de ses principes - n'est pas sans récompenses. Elle côtoie des vedettes de première : George Clooney, $\mathrm{Me}$ lanie Griffith, Brad Pitt (sans Angelina), Leonardo DiCaprio, Sophie Marceau, Bill Clinton, et un Bill Gates étonnamment 
priapique. En termes de charme, elle estime que « Bob » De Niro est sans égal. Comme les autres textes de Salvayre, ce livre est intelligent et acerbe, à la fois amusant et inquiétant. Salvayre déploie la satire de façon multiple, mais surtout pour nous faire réfléchir sur l'art et le pouvoir, et plus précisément encore sur la manière qu'a celui-ci de l'emporter sur celui-là, le pouvoir persuadant l'art de se mettre sur le dos, afin de se faire caresser le ventre.

La satire est encore une fois l'outil de choix de Salvayre dans Petit Traité de l'éducation lubrique (2008). C'est un livre qui fête la sexualité humaine, animé par le désir et l'humour. Proposé comme un manuel destiné aux illettrés sexuels, le livre passe en revue une multiplicité de pratiques, avec un tact admirable. Son langage est extrêmement classique, châtié, un langage qu'on aurait pu croire inadapté pour certains actes parmi les plus vigoureux que Salvayre décrit. Mais c'est bien cela l'intérêt, car les tensions qu'elle construit contribuent de manière importante aux effets qu'elle cherche à produire. S'efforçant d'édifier les plus désespérément naïfs entre nous, Salvayre vise en fait les puritains, les gourous, les mollahs, les flics sexuels, et les gens rangés et crispés de toutes sortes, visant un vaste horizon avec le lanceflammes de son regard satirique. C'est un petit livre bien rafraîchissant.

Dans BW (2009) le talent de portraitiste de Salvayre se fait encore sentir, mais cette fois-ci elle peint d'après nature. Son sujet, c'est Bernard Wallet, son compagnon de longue date. Wallet (dont vous lirez le « Lydie Salvayre, écrivain baroque'n'roll » dans le volume présent) est un homme hors du commun, une figure rocambolesque qui aurait pu être un héros de roman, s'il n'avait pas été si absolument réel. Athlète au niveau olympique dans sa jeunesse, voyageur invétéré, observateur lucide de tendances idéologiques, fervent 
défenseur de la culture, Wallet a passé la majorité de sa vie dans le monde de l'édition. Il fonde les Éditions Verticales en 1997, où il publie des écrivains comme Yves Pagès, Gabrielle Wittkop, Olivia Rosenthal, Pierre Senges, Nicole Caligaris, Régis Jauffret, Maylis de Kerangal et Salvayre elle-même. Il quitte Verticales en 2008, et la même année il perd sa vision pendant un temps. C'est cette calamité qui inspire $B W$, où Salvayre inscrit ce que Wallet a besoin de dire à propos de sa vie, motivé par l'idée d'une cécité permanente. Aveugle, Wallet s'efforce de voir les choses en face, une posture que Salvayre partage sans compromis.

Dans les premières pages d'Hymne (2011) Salvayre remarque qu'elle ressent le passage du temps, et qu'elle a par conséquent envie d'écrire désormais seulement sur les choses qui la touchent le plus profondément, sur les œuvres qu'elle admire infiniment. Ici, l'œuvre en question, c'est le « Star-Spangled Banner » que Jimi Hendrix a joué à Woodstock, le 18 août 1969. Pour Salvayre, cette performance constitue un événement exceptionnel, quelque chose qui a marqué son moment, mais qui sert aussi à poser les termes d'une vision neuve. Sa perspective est parfois très personnelle, parfois historique, politique, sociale ou culturelle ; mais quel que soit le caractère de son regard, son analyse est toujours pénétrante. Elle voit beaucoup de choses différentes dans l'hymne national réinventé. L'articulation d'un « cri insoutenable » plus puissant que les mots ; un geste qui demeure totalement radical, même après quatre décennies; un «sanglot terrible » qui déchire l'espace qu'il libère. C'est à la fois courageux et intransigeant. Cela nie de façon directe la notion d'une Amérique unie et indivisée, la décrivant plutôt comme une pluralité, et proposant l'idée même de la pluralité comme force créatrice. Selon Salvayre, il s'agit d'une chose aussi belle que le dernier 
mouvement de la neuvième symphonie de Beethoven, aussi puissante que Guernica de Picasso. C'est une chose catégoriquement particulière et inimitable. La performance de Hendrix, dans son éloquence pure, nourrit l'imagination de Salvayre et l'encourage, à son tour, à mettre son propre talent d'artiste en œuvre.

Dans 7 Femmes (2013) Salvayre désigne sept écrivaines qui comptent beaucoup pour elle : Emily Brontë, Djuna Barnes, Sylvia Plath, Colette, Marina Tsvetaeva, Virginia Woolf et Ingeborg Bachmann. Pour chacune d'elles, la notion d'une vie simple ne suffisait pas, selon Salvayre. Elles ont toutes refusé l'ordre établi des choses, chacune choisissant d'inscrire ce refus dans leur écriture. Certaines ont hurlé et fait claquer les portes en écrivant ; d'autres ont écrit avec plus de grâce étudiée. Mais chacune en est venue à parler d'une voix tout à fait particulière et reconnaissable. Encore plus remarquable aux yeux de Salvayre est le fait que dans chaque cas l'œuvre s'est confondu avec la vie, sans égard pour les sacrifices nécessaires. Salvayre voit de la folie dans une telle décision, mais la passion qui la sous-tend l'intéresse bien davantage. Elle remarque qu'elle a relu les livres de ces femmes - tous les livres de ces femmes - pendant une période où elle trouvait l'écriture impossible. De toute évidence il y avait quelque chose dans cette expérience qui a servi à attiser sa propre créativité, résultant dans ce livre ainsi que d'autres à sa suite.

Le Prix Goncourt décerné à Lydie Salvayre pour Pas pleurer (2014) lui a valu un lectorat élargi, ainsi qu'une attention médiatique très considérable. Dans ce roman, Salvayre se penche sur certains de ses thèmes les plus chers : la dynamique de la libération personnelle, par exemple, et surtout la libération de la femme ; une fascination pour l'histoire et pour la manière dont le passé conditionne notre expérience 
du présent ; la volonté d'examiner les gestes spécifiques de la révolte ; le désir de donner voix à des gens qui, approchant la fin de la vie, se demandent ce qui leur est arrivé en chemin. Montse, la mère de la narratrice, se trouve au centre du récit. À l'âge de quinze ans, en pleine Guerre Civile, elle avait quitté sa maison natale afin de rejoindre les forces républicaines à Barcelone. Ce qu'elle a vécu l'a transformée, à deux niveaux différents. D'une part (et dans une perspective sociale et politique), elle a été fortement impressionnée par la ville révolutionnaire, et surtout par la ferveur révolutionnaire spécifiquement anarchique si largement répandue à Barcelone à l'époque. D'autre part (dans un registre plus personnel et intimiste), elle a été galvanisée par l'expérience sexuelle qu'elle y a connue, dont l'effet était aussi immédiat que profond. Dans le « maintenant » narratif du récit, Montse a quelques quatre-vingt-dix ans, et sa mémoire faiblit. Ses expériences de jeunesse lui semblent plus proches que tout ce qui lui est arrivé depuis, et elle parle à sa fille comme si le reste de sa vie ne comptait pas pour grandchose. Elle a abandonné tout ce qui lui paraît inessentiel ou supplémentaire afin de se concentrer sur les événements de sa vie qui lui semblent fondamentaux. Salvayre attire notre attention sur ce processus de vannage, suggérant que si cela vaut pour la vie, cela vaut également pour l'art.

Tout homme est une nuit (2017) aborde le thème de l'étranger, racontant l'histoire d'un homme qui arrive dans un petit village, inconnu et imprévu, pour s'y établir. Il souffre d'un cancer, et il cherche un endroit où se reposer. Au lieu de cela, c'est la malveillance qui l'attend, et la suspicion, et même la haine. Les villageois imaginent le pire à son sujet : c'est sans doute un monstre sexuel, ou un trafiquant de drogues dures, ou alors un Musulman. C'est la dynamique du groupe qui intéresse Salvayre, la manière dont 
le groupe se définit par opposition aux étrangers (sans qui le groupe n'aurait ni logique ni raison d'être). Le processus de diabolisation qu'elle décrit est terrifiant - et pourtant certains de ses lecteurs et lectrices reconnaîtront cela comme faisant partie du réel vécu. Cependant, de même que le groupe interroge l'étranger, l'étranger met le groupe en procès. Ces gestes ne sont pas parfaitement équilibrés certes, parce que les uns détiennent plus de pouvoir et d'influence que les autres. Tout de même, la subversion et la force de la résistance se rangent du côté de l'étranger, et ce sont deux principes qui fascinent Salvayre, depuis toujours.

Marcher jusqu'au soir (2019), son livre le plus récent au moment où j'écris ceci (et dont elle offre quelques extraits dans ce volume), résulte d'une invitation de passer toute une nuit, seule, au Musée Picasso, à Paris. S'il est largement question de Picasso encore une fois, il s'agit aussi d'Alberto Giacometti, car la visite de Salvayre a eu lieu pendant l'exposition Picasso-Giacometti organisé par le musée. Salvayre marche dans les couloirs du musée, contemplant L'Homme qui marche de Giacometti, cette construction si saisissante de notre frêle humanité. À quoi est-ce que cela sert, l'œuvre d'art, même (et peut-être surtout) lorsqu'il s'agit d'un geste aussi immense que celui de Giacometti. Qu'est-ce que nous devons faire de cela, pour qui est-ce, comment est-ce que nous pouvons le comprendre, quel sens est-ce que cela articule par rapport à notre vie, et d'ailleurs quelle vie est la nôtre, justement ? Voilà les questions que Salvayre se pose, et pas pour la première fois, car d'une manière ou d'autre ce sont des questions qu'elle invoque sans cesse depuis le début de sa carrière.

Prenant un pas en arrière et considérant cette carrière avec un peu de distance, un lecteur pourrait conclure que Lydie Salvayre a choisi de travailler là où l'expérience humaine 
rentre dans le vif du vivant. Dans ces moments où notre juste colère éclate, ou notre désir, notre deuil, notre indignation, notre peur, notre aspiration, notre pitié, notre espoir. Salvayre écrit dans l'urgence, soulignant des choses que nous avons ignorées mais qui nous concernent directement, soit individuellement, soit collectivement. Au début de cet essai j'ai suggéré que ses livres se jouent contre le grain de la société contemporaine, que nous pouvons les imaginer comme des antidotes contre les bêlements de ceux qui cherchent à nous contrôler et les flagorneries transparentes de ceux qui cherchent à nous séduire. Je suis persuadé que les livres de Salvayre peuvent nous aider à reconnaître certaines forces pernicieuses qui nous confrontent, et à y résister. Pourtant, ces livres nous offrent bien plus que cela, et je n'ai peut-être pas assez insisté sur la remarquable joie qui les anime, sur leur exubérance, sur la générosité d'esprit qu'on trouve dans chacun. Ce sont des effets hospitaliers qui servent à nous accueillir dans les mondes que ces livres esquissent, qui suggèrent que nous y sommes bienvenus, qu'il y a justement une place pour nous dans ces mondes. 